\title{
Long-term statin use before primary percutaneous coronary intervention improves treatment outcomes of acute myocardial infarction
}

\author{
RUIWEI GUO ${ }^{1}$, LIXIA YANG $^{1}$, LIHUA MU $^{2}$, XIANFENG PAN $^{3}$ and FENG QI ${ }^{1}$ \\ ${ }^{1}$ Department of Cardiology, Kunming General Hospital of Chengdu Military Command, Kunming, Yunnan 650032; \\ ${ }^{2}$ Department of Emergency Medicine, Yunnan Provincial Emergency Center, Kunming, Yunnan 650000 ; ${ }^{3}$ Department of \\ Emergency Medicine, Kunming General Hospital of Chengdu Military Command, Kunming, Yunnan 650032, P.R. China
}

Received August 31, 2015; Accepted October 19, 2016

DOI: $10.3892 /$ etm.2017.4156

\begin{abstract}
Numerous studies have reported that high-dose statin loading therapy prior to primary percutaneous coronary intervention (PPCI) improves the clinical outcomes of patients following acute myocardial infarction (AMI). However, little is known about the effects of long-term statin use prior to PPCI on such outcomes. Therefore, the aim of the present analysis was to clarify the effects of long-term statin use before PPCI on the treatment outcomes of patients following AMI. The records of 213 patients who had AMI and met the inclusion criteria were retrospectively reviewed. Patients were divided into two groups: A control group $(n=178)$ who had received no statin pretreatment before AMI onset, and a statin group $(n=35)$ who had received statin treatment for $\geq 1$ month before AMI onset. All patients received a standard treatment regimen for the secondary prevention of coronary artery disease after PPCI. Baseline clinical variables, details of the PPCI procedure and clinical outcomes within 3 months after treatment were reviewed. Patients in the statin group were significantly older than those in the control group $(\mathrm{P}=0.003)$. Compared with the control group, there was a greater proportion of patients with hyperlipidemia and previous angina pectoris in the statin group. There were no differences in the use of other drugs (aspirin, $\beta$-blockers and angiotensin-converting enzyme inhibitors) prior to PPCI between the two groups. The corrected TIMI frame count (cTFC) was significantly lower in the statin group than in the control group $(24.1 \pm 12.8$ vs. $29.4 \pm 14.3$, respectively; $\mathrm{P}=0.043$ ). Multivariable linear regression analysis showed that long-term statin use before AMI was a significant predictor of cTFC after PPCI $(\mathrm{P}=0.012)$.
\end{abstract}

Correspondence to: Dr Lixia Yang, Department of Cardiology, Kunming General Hospital of Chengdu Military Command, 212 Daguan Road, Kunming, Yunnan 650032, P.R. China

E-mail: grw1977@hotmail.com

Key words: statin, acute myocardial infarction, primary percutaneous coronary intervention, clinical outcomes
Furthermore, the incidence of major adverse cardiac events within 3 months after PPCI was higher in the control group than in the statin group (16.8 vs. $2.9 \%$, respectively; $\mathrm{P}=0.032$ ). Logistic regression analysis showed that previous statin use was associated with the incidence of major adverse cardiac events within 3 months after treatment $(\mathrm{P}=0.012)$. The results of the present study demonstrate that long-term statin use prior to PPCI improved treatment outcomes after AMI in actual clinical practice.

\section{Introduction}

Treatment outcomes of patients after acute myocardial infarction (AMI) have greatly improved following the introduction of primary percutaneous coronary intervention (PPCI) (1). However, major adverse cardiac events (MACEs) continue to occur at markedly higher rates in patients after AMI as compared with those with stable coronary artery disease, particularly within the first 3 months after PPCI (2).

It is well established that statin treatment is beneficial for the primary and secondary prevention of coronary artery diseases $(3,4)$. In addition to lowering serum lipid levels, statins have been shown to convey favorable effects on endothelial function, coagulation activation, nitric oxide bioavailability and stimulation of the inflammatory response. Collectively, these effects are known as the 'pleiotropic effects' of statins (5). Recently, the 'pleiotropic effects' of statins have also been shown to modulate platelet adhesion and activation, inhibit inflammation, and attenuate thrombosis, all of which may contribute to the preservation of microvascular function during ischemia and reperfusion following ST-elevation myocardial infarction (STEMI) (6-8). Several studies have demonstrated that high-dose statin loading therapy prior to PPCI may be more efficacious in patients with STEMI than in other clinical situations because STEMI is characterized by extreme inflammation (9-11). However, little is known about the long-term effects of statin loading prior to PPCI on treatment outcomes of patients after AMI.

A recent meta-analysis indicated a time-related impact of statin therapy on the clinical outcomes of patients with acute coronary syndrome (ACS) undergoing PPCI: The earlier the 
administration before PPCI, the greater the benefits. Earlier statin administration was found to be significantly correlated with a lower risk of AMI, MACEs and major adverse cardiac and cerebrovascular events within 30 days after treatment (12). Previous observational studies of patients after STEMI have suggested that previous chronic statin use may improve coronary blood flow and is associated with reduced short-term mortality (13). Based on the findings of these studies, it may be hypothesized that long-term statin use prior to PPCI could improve the treatment outcomes of patients after AMI. Therefore, the present retrospective study was performed to clarify the effects of long-term statin use prior to PPCI on the treatment outcomes of patients after AMI.

\section{Materials and methods}

Study population. The medical records of 268 patients who underwent PPCI for AMI at the Kunming General Hospital of Chengdu Military Command (Kunming, China) from January 2014 to March 2015 were retrospectively reviewed. Of these, 55 patients were excluded because of incomplete follow-up data; thus, the study population consisted of 213 patients. For the purposes of the present study, patients were divided into two groups according to statin treatment for at $\geq 1$ month prior to AMI onset. A total of 35 patients received statin treatment $(20 \mathrm{mg}$ atorvastatin/day or $10 \mathrm{mg}$ rosuvastatin/day) before AMI onset because of hyperlipidemia or coronary artery disease (statin group). The other 178 patients did not receive statin pretreatment before AMI onset (control group). All patients received standard treatment for secondary prevention of coronary artery diseases after PPCI, including treatment with aspirin, clopidogrel, ACE inhibitors, beta blockers and statins. The following patient information was collected: i) Patient age; ii) gender; iii) risk factors for cardiovascular disease, including diabetes (fasting plasma glucose and two-hour plasma glucose value of $\geq 126 \mathrm{mg} / \mathrm{dl}$ and $\geq 200 \mathrm{mg} / \mathrm{dl}$, respectively), hypertension (a systolic blood pressure $\geq 140 \mathrm{mmHg}$ or a diastolic blood pressure $\geq 90 \mathrm{mmHg}$ ), hyperlipidemia (a total cholesterol level $\geq 240 \mathrm{mg} / \mathrm{dl}$ ), smoking history and previous angina pectoris; iv) previous medications prior to PPCI; v) the time from chest pain to balloon dilation (PTB) were measured according to inquiry and standard procedure; vi) details of PPCI treatment; and vii) myocardial infarct outcomes within 3 months after treatment. All patients provided informed consent, and the study protocol was approved by the Medical Ethics Committee of Kunming General Hospital of Chengdu Military Command (approval no. 2015067).

PPCI procedure. After angiography, the PPCI procedure was performed using standard techniques by an experienced operator. Prior to the procedure, $300 \mathrm{mg}$ aspirin (Bayer AG, Leverkusen, Germany) and $600 \mathrm{mg}$ clopidogrel (Sanofi S.A., Paris, France) were administered to all patients. An intravenous bolus of 5,000 units heparin (Kunming Jida Pharmaceutical Co., Ltd, Kunming, China) was administered to maintain an activated clotting time of $>300 \mathrm{sec}$ during PPCI. Culprit vessel blood flow was evaluated using thrombolysis in myocardial infarction (TIMI) grade and corrected TIMI frame count (cTFC). Specific details regarding TIMI flow grades and cTFC have been described previously (14). TIMI flow grades and cTFC were evaluated following the completion of PPCI.
Study end point. The primary end point of the study was the incidence of MACEs within 3 months after treatment, including all-cause mortality rates, the incidence of new myocardial infarction, and target vessel revascularization (TVR). New myocardial infarction and TVR were defined as previously described (15). Only the first MACE was considered. The secondary end points included TIMI flow grade and cTFC after PPCI.

Statistical analysis. Continuous variables are presented as means \pm standard deviations and were compared using the t-test in cases of two independent groups with parametric data. Categorical variables are presented as frequencies with percentages, and comparisons were made using the Pearson chi-squared test. Multiple linear regression analysis was performed to determine the influence of previous statin treatment on cTFC after PPCI. The following variables were entered into the model: Age, gender, hypertension, diabetes, hypercholesterolemia, smoking history, previous angina pectoris, drugs given before AMI onset, PTB time, multivessel disease, and stent length and diameter. A multivariable logistic regression model was constructed to predict the occurrence of MACEs within 3 months after PPCI. The following variables were selected and inserted into the logistic regression analysis: Age, gender, hypertension, diabetes, hypercholesterolemia, smoking history, previous angina pectoris, peak creatine kinase concentration, drugs given before AMI onset, PTB time, multivessel disease, stent length and diameter, and cTFC. A two-tailed probability $(\mathrm{P})$-value of $<0.05$ was considered to indicate a statistically significant difference. All analyses were performed using SPSS version 19.0 software (IBM SPSS, Armonk, NY, USA).

\section{Results}

Baseline characteristics. The key baseline characteristics of patients eligible for the study are listed in Table I. A total of 35 patients received long-term statin administration (28 patients with $20 \mathrm{mg}$ atorvastatin/day and 7 patients with $10 \mathrm{mg}$ rosuvastatin/day) for $>1$ month before AMI onset. Of these patients, 34 received long-term statin administration to treat hyperlipidemia and the other received this treatment because of previous angina pectoris. Patients in the statin group were significantly older than those in the control group (67.3 \pm 4.9 vs. $61.8 \pm 10.4$ years, respectively; $\mathrm{P}=0.003)$. Compared with the control group, hyperlipidemia and previous angina pectoris were more prevalent in the statin group (97.1 vs. $29.2 \%$, respectively; $\mathrm{P}<0.001$; and 25.7 vs. $10.1 \%$, respectively; $\mathrm{P}=0.011)$. There were no significant differences in gender, diabetes, hypertension, smoking history, PTB time or peak creatine kinase concentrations between the groups. Furthermore, there were no differences in the frequency of administration of other drugs (i.e., aspirin, B-blockers and angiotensin-converting-enzyme inhibitors) prior to PPCI between the two groups.

PPCI procedure and clinic outcomes within 3 months. All patients underwent angiography and PPCI. Clinical outcomes of PPCI are listed in Table II. Glycoprotein IIb/IIIa platelet inhibitors and thrombectomy devices were used when a 
Table I. Baseline clinical characteristics.

\begin{tabular}{|c|c|c|c|}
\hline Characteristic & Control group (n=178) & Statin group $(n=35)$ & P-value \\
\hline Age, years ${ }^{\mathrm{a}}$ & $61.8 \pm 10.4$ & $67.3 \pm 4.9^{b}$ & 0.003 \\
\hline Male, n (\%) & $131(73.6)$ & $25(71.4)$ & 0.791 \\
\hline Peak creatine kinase (U/l) & $1,794.1 \pm 1072.1$ & $1,670.6 \pm 890.1$ & 0.523 \\
\hline \multicolumn{4}{|l|}{ Risk factor, n (\%) } \\
\hline Diabetes & $48(27.0)$ & $13(37.1)$ & 0.796 \\
\hline Hypertension & $62(34.5)$ & $14(40.0)$ & 0.560 \\
\hline Hyperlipidemia & $52(29.2)$ & $34(97.1)^{b}$ & $<0.001$ \\
\hline Smoking history & $47(26.4)$ & $12(34.3)$ & 0.341 \\
\hline Previous angina pectoris & $18(10.1)$ & $9(25.7)^{\mathrm{b}}$ & 0.011 \\
\hline \multicolumn{4}{|l|}{ Previous medications, $\mathrm{n}(\%)$} \\
\hline Aspirin & $26(14.6)$ & $7(20.0)$ & 0.420 \\
\hline$\beta$-blocker & $7(3.9)$ & $3(8.6)$ & 0.236 \\
\hline ACEI & $13(7.3)$ & $6(17.1)$ & 0.062 \\
\hline PTB time (h) & $9.1 \pm 3.3$ & $19.6 \pm 4.2$ & 0.584 \\
\hline
\end{tabular}

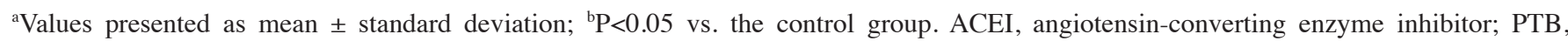
pain-to-balloon.

Table II. Primary percutaneous coronary intervention management and clinical outcomes.

\begin{tabular}{|c|c|c|c|}
\hline Management and outcomes & Control group $(\mathrm{n}=178)$ & Statin group $(n=35)$ & P-value \\
\hline Multivessel disease, $\mathrm{n}(\%)$ & $68(38.2)$ & $19(54.3)$ & 0.077 \\
\hline \multicolumn{4}{|l|}{ Culprit vessel } \\
\hline Left main & $1(0.6)$ & $0(0.0)$ & 0.657 \\
\hline Left anterior descending & $79(44.4)$ & $15(42.9)$ & 0.868 \\
\hline Left circumflex & $26(14.6)$ & $6(17.1)$ & 0.701 \\
\hline Right coronary artery & $72(40.4)$ & $14(40.0)$ & 0.960 \\
\hline Use of glycoprotein inhibitor, n (\%) & $23(12.3)$ & $6(17.1)$ & 0.506 \\
\hline Use of thrombectomy devices, n (\%) & $10(5.6)$ & $4(11.4)$ & 0.205 \\
\hline Length of stents $(\mathrm{mm})^{\mathrm{a}}$ & $27.6 \pm 8.0$ & $25.9 \pm 8.8$ & 0.255 \\
\hline Diameter of stents $(\mathrm{mm})^{\mathrm{a}}$ & $3.3 \pm 0.3$ & $3.2 \pm 0.3$ & 0.193 \\
\hline Final TIMI 3 flow, n (\%) & $161(90.4)$ & $32(91.4)$ & 0.856 \\
\hline $\mathrm{cTFC}^{\mathrm{a}}$ & $29.4 \pm 14.3$ & $24.1 \pm 12.8^{b}$ & 0.043 \\
\hline \multicolumn{4}{|l|}{ Clinical outcome at 3 months, n (\%) } \\
\hline All cause mortality & $8(4.5)$ & $1(2.9)$ & 0.660 \\
\hline New myocardial infarction & $6(3.4)$ & $1(2.9)$ & 0.876 \\
\hline TVR & $22(12.3)$ & $1(2.9)$ & 0.088 \\
\hline MACEs & $30(16.8)$ & $1(2.9)^{\mathrm{b}}$ & 0.032 \\
\hline
\end{tabular}

${ }^{a}$ Values presented as mean \pm standard deviation; ${ }^{b} \mathrm{P}<0.05$ vs. the control group. MACEs includes all-cause mortality, new myocardial infarction and TVR. TIMI, thrombolysis in myocardial infarction; cTFC, corrected TIMI frame count; TVR, target vessel revascularization; MACEs, major adverse cardiac events.

large number of thrombi were observed. The cTFC value was significantly lower in the statin group than in the control group ( $24.1 \pm 12.8$ vs. $29.4 \pm 14.3$, respectively; $\mathrm{P}=0.043$ ). There were no significant differences in other angiographic or procedural outcomes between groups. The 3-month clinical outcomes are listed in Table II. There were no significant differences in all-cause mortality rates $(\mathrm{P}=0.660)$ or in the incidence of new myocardial infarction $(\mathrm{P}=0.876)$ between the two groups. However, there was tendency toward a higher rate of TVR among patients in the control group (12.3 vs. $2.9 \%$, respectively; $\mathrm{P}=0.088)$. This tendency may underlie the higher prevalence of major adverse cardiac events in the 
Table III. Multivariate linear regression determinants of cTFC after PCI.

\begin{tabular}{lcccc}
\hline Determinant & $\mathrm{B}$ & $\mathrm{t}$ & $95 \%$ CI & P-value \\
\hline Previous statin use & 7.920 & 2.547 & $1.787-14.054$ & 0.012 \\
Age & 0.267 & 2.552 & $0.610-0.474$ & 0.011 \\
Diabetes & 4.802 & 2.210 & $0.443-9.161$ & 0.031 \\
PTB times & 0.631 & 0.244 & $0.149-1.112$ & 0.011
\end{tabular}

Variables entered for the multivariate linear regression analyses were age, gender, hypertension, diabetes, hypercholesterolemia, smoking, previous angina pectoris, drugs given before the onset of acute myocardial infarction, PTB times, multivessel disease, and the length and diameter of stents. cTFC, corrected thrombolysis in myocardial infarction frame count; PCI, percutaneous coronary intervention; PTB, pain-to-balloon; CI, confidence interval.

Table IV. Multivariate logistic regression determinants of 3-month MACEs after PCI.

\begin{tabular}{|c|c|c|c|c|}
\hline Determinant & $\mathrm{B}$ & OR & $95 \% \mathrm{CI}$ & P-value \\
\hline Previous statin use & 2.694 & 14.788 & $1.023-213.870$ & 0.048 \\
\hline Age & 0.100 & 1.105 & $1.027-1.190$ & 0.008 \\
\hline Diabetes & -1.896 & 0.150 & $0.030-0.744$ & 0.020 \\
\hline Hypertension & -1.646 & 0.193 & $0.048-0.769$ & 0.020 \\
\hline Smoking history & 1.652 & 5.216 & $1.525-17.840$ & 0.008 \\
\hline PTB times & 0.149 & 1.543 & $0.261-9.141$ & 0.033 \\
\hline Peak creatine kinase & 0.001 & 1.001 & $1.000-1.001$ & 0.007 \\
\hline Diameter of stent & -2.238 & 0.107 & $0.014-0.795$ & 0.029 \\
\hline cTFC & 0.070 & 1.073 & $1.034-1.113$ & $<0.001$ \\
\hline
\end{tabular}

Variables entered for the multivariable logistic regression analyses were age, gender, hypertension, diabetes, hypercholesterolemia, smoking, previous angina pectoris, peak creatine kinase, drugs given before the onset of acute myocardial infarction, PTB times, multivessel disease, length and diameter of stents, and cTFC. MACEs, major adverse cardiac events; PCI, percutaneous coronary intervention; PTB, pain-to-balloon; cTFC, corrected thrombolysis in myocardial infarction frame count; CI, confidence interval; OR, odds ratio.

control group compared with the statin group (16.8 vs. $2.9 \%$, respectively; $\mathrm{P}=0.032)$.

Association between long-term statin use before AMI and $c T F C$. Multivariable linear regression analysis was performed to assess the effects of long-term statin use before AMI on cTFC after PPCI. The multivariate regression determinants of cTFC during PPCI are shown in Table III. The regression model showed that long-term statin use before AMI was a significant predictor of cTFC after PPCI $(\mathrm{P}=0.012)$. Other independent predictors of cTFC after PPCI were patient age $(\mathrm{P}=0.011)$, presence of diabetes $(\mathrm{P}=0.031)$, and $\mathrm{PTB}$ time $(\mathrm{P}=0.011)$.

Association between long-term statin use before AMI and MACEs within 3 months after treatment. Multivariate logistic regression analysis showed that previous statin use was associated with MACEs within 3 months (Table IV; $\mathrm{P}=0.048$ ). Other factors significantly associated with MACEs within 3 months after treatment included patient age $(\mathrm{P}=0.008)$, diabetes $(\mathrm{P}=0.020)$, hypertension $(\mathrm{P}=0.020)$, smoking history $(\mathrm{P}=0.008), \mathrm{PTB}$ time $(\mathrm{P}=0.033)$, peak creatine kinase concentration $(\mathrm{P}=0.007)$, stent diameter $(\mathrm{P}=0.029)$ and $\mathrm{cTFC}$ $(\mathrm{P}<0.001)$.

\section{Discussion}

Statin use is acknowledged to be beneficial to patients after AMI through 'pleiotropic mechanisms' additional to the ability of the treatment to lower low-density lipoprotein cholesterol levels $(4,5)$. The results of the present study demonstrated that long-term statin use prior to AMI improves clinical outcomes after PPCI, despite a substantially higher risk profile in the statin group. In addition, the improved coronary reflow after PPCI, as assessed by cTFC, may be attributable to the beneficial outcomes observed in the statin group. These conclusions are based on several lines of evidence. First, the cTFC value was significantly lower in the statin group than in the control group. Second, the regression model showed that long-term statin use before AMI was a significant predictor of cTFC after PPCI. Third, multivariate logistic regression analysis showed that previous statin use was also associated with the occurrence of MACEs within 3 months. In addition, some predictors of clinical outcomes within 3 months after PPCI were identified.

The results of this retrospective study revealed significantly greater risk factors among patients in the statin group, specifically, age, hyperlipidemia and previous angina pectoris. These results are in accordance with those of some previous 
trials (16-18). The main reason for a greater risk of MACEs in older people is that the risk of coronary heart disease is greater among the elderly and those patients at greater risk are more likely to reject statin treatment due to associated liver toxicity. In order to analyze the effects of long-term statin use before AMI after PPCI, cTIMI frame count was employed in this study because it is a simple clinical tool for the assessment of quantitative indices of coronary blood flow (19). In addition, cTFC is closely associated with the clinical outcomes of patients after AMI (20). The results of the present study showed that cTFC values were significantly lower in the statin group than in the control group, and the regression model indicated that long-term statin use before AMI was a significant predictor of cTFC following PPCI. Celik et al (6) also reported that prior statin use may improve coronary blood flow after PPCI in patients after AMI, possibly due to its beneficial effects on microvascular function. However, another study reported that atorvastatin loading may not convey protective effects on endothelial function or against inflammatory responses in patients with STEMI undergoing primary PPCI (5). These results indicate that long-term statin use may have favorable effects on microvascular function. Another previous study showed that chronic statin administration preserved coronary microvascular integrity independent of lipid-lowering effects (21).

There is considerable evidence to indicate that statin therapy improves the clinical outcomes of patients with ACS undergoing PPCI. The ARMYDA-ACS trial, which was the first randomized study to assess the efficacy of statin therapy prior to PPCI in patients with ACS, showed that loading with $80 \mathrm{mg}$ atorvastatin $12 \mathrm{~h}$ before PPCI reduced the elevation in post-procedural biomarkers and the incidence of MACEs within 30 days after treatment (22). The Euro Heart Survey trial reported a reduction in all-cause 7-day mortality in patients with ST-elevation ACS who received statin treatment within $24 \mathrm{~h}$ after admission, as compared with patients who did not receive statins within the first $24 \mathrm{~h}$ (23). The current study also evaluated the effect of long-term statin use prior to PPCI on the clinical outcomes of patients after AMI. The results of multivariate logistic regression analysis revealed that previous statin use was associated with a lower incidence of MACEs within 3 months after treatment. Moreover, Lev et al (16) also reported that previous statin therapy in patients who underwent PPCI after STEMI may be associated with reduced short-term mortality. Lee et al (24) demonstrated that early and continuous statin therapy can improve the early outcomes of STEMI patients following PPCI in actual clinical practice. Since these cited reports were retrospective studies, a large prospective study is required to confirm the effect of long-term statin use prior to PPCI on the clinical outcomes of patients after AMI.

There were several limitations to the present study that should be addressed. First, this study was retrospective and there were large differences in baseline clinical characteristics between the two patient groups, particularly age and risk profiles. Second, there was no control for statin properties or dosage among patients prior to PPCI. Third, this study was a single-center study; thus the data may not be representative of other institutions. In addition, the sample size of the study was relatively small. Therefore, a larger cohort is needed to confirm the study findings.
In conclusion, the results of the present study demonstrated that long-term statin use prior to PPCI improved the treatment outcomes of patients after AMI in actual clinical practice.

\section{Acknowledgements}

This study was supported by the Yunnan Province Foundation (grant. no. 2014HB035) and the China Scholarship Council (grant no. 201407820113).

\section{References}

1. Komócsi A, Aradi D, Kehl D, Ungi I, Thury A, Pintér T, Di Nicolantonio JJ, Tornyos A and Vorobcsuk A: Meta-analysis of randomized trials on access site selection for percutaneous coronary intervention in ST-segment elevation myocardial infarction. Arch Med Sci 10: 203-212, 2014.

2. Lee YH, Park JS, Tahk SJ, Hwang GS, Yoon MH, Choi SY, Choi BJ, Lim HS, Yang HM, Seo KW and Shin JH: $\beta$-blocker therapy in the era of primary percutaneous intervention for ST elevation myocardial infarction. Cardiology 132: 91-100, 2015 (Epub ahead of print).

3. Räber L, Taniwaki M, Zaugg S, Kelbæk H, Roffi M, Holmvang L, Noble S, Pedrazzini G, Moschovitis A, Lüscher TF, et al: Effect of high-intensity statin therapy on atherosclerosis in non-infarct-related coronary arteries (IBIS-4): A serial intravascular ultrasonography study. Eur Heart J 36: 490-500, 2015.

4. Athyros VG, Katsiki N, Karagiannis A and Mikhailidis DP: Short-, mid-, and long-term benefits of peri-procedural high-intensity statin administration in patients undergoing percutaneous coronary intervention. Curr Med Res Opin 31: 191-195, 2015.

5. Yong H, Wang X, Mi L, Guo L, Gao W, Zhang Y and Cui M: Effects of atorvastatin loading prior to primary percutaneous coronary intervention on endothelial function and inflammatory factors in patients with ST-segment elevation myocardial infarction. Exp Ther Med 7: 316-322, 2014.

6. Celik T, Kursaklioglu H, Iyisoy A, Kose S, Kilic S, Amasyali B, Kardesoglu E and Isik E: The effects of prior use of atorvastatin on coronary blood flow after primary percutaneous coronary intervention in patients presenting with acute myocardial infarction. Coron Artery Dis 16: 321-326, 2005.

7. Leoncini M, Toso A, Maioli M, Tropeano F and Bellandi F: Statin treatment before percutaneous cononary intervention. J Thorac Dis 5: 335-342, 2013.

8. Shen W, Shi HM, Fan WH, Luo XP, Jin B and Li Y: The effects of simvastatin on angiogenesis: Studied by an original model of atherosclerosis and acute myocardial infarction in rabbit. Mol Biol Rep 38: 3821-3828, 2011.

9. Kim JW, Yun KH, Kim EK, Kim YC, Joe DY, Ko JS, Rhee SJ, Lee EM, Yoo NJ, Kim NH, et al: Effect of high dose rosuvastatin loading before primary percutaneous coronary intervention on infarct size in patients with ST-segment elevation myocardial infarction. Korean Circ J 44: 76-81, 2014.

10. Benjo AM, El-Hayek GE, Messerli F, DiNicolantonio JJ, Hong MK, Aziz EF, Herzog E and Tamis-Holland JE: High dose statin loading prior to percutaneous coronary intervention decreases cardiovascular events: A meta-analysis of randomized controlled trials. Catheter Cardiovasc Interv 85: 53-60, 2015.

11. Jang Y, Zhu J, Ge J, Kim YJ, Ji C and Lam W: Preloading with atorvastatin before percutaneous coronary intervention in statin-naïve Asian patients with non-ST elevation acute coronary syndromes: A randomized study. J Cardiol 63: 335-343, 2014.

12. Navarese EP, Kowalewski M, Andreotti F, van Wely M, Camaro C, Kolodziejczak M, Gorny B, Wirianta J, Kubica J, Kelm M, et al: Meta-analysis of time-related benefits of statin therapy in patients with acute coronary syndrome undergoing percutaneous coronary intervention. Am J Cardiol 113: 1753-1764, 2014.

13. Eisen A, Leshem-Lev D, Yavin H, Orvin K, Mager A, Rechavia E, Bental T, Dadush O, Battler A, Kornowski R and Lev EI: Effect of high dose statin pretreatment on endothelial progenitor cells after percutaneous coronary intervention (HIPOCRATES Study). Cardiovasc Drugs Ther 29: 129-135, 2015. 
14. Bickel C, Rupprecht HJ, Maimaitiming A, Welk I, Blankenberg S, Krummenauer $\mathrm{F}$ and Meyer J: The superiority of TIMI frame count in detecting coronary flow changes after coronary stenting compared to TIMI Flow Classification. J Invasive Cardiol 14: 590-596, 2002.

15. Alfonso F, Pérez-Vizcayno MJ, Cárdenas A, García Del Blanco B, García-Touchard A, López-Minguéz JR, Benedicto A, Masotti M, Zueco J, Iñiguez A, et al: A prospective randomized trial of drug-eluting balloons versus everolimus-eluting stents in patients with in-stent restenosis of drug-eluting stents: The RIBS IV randomized clinical trial. J Am Coll Cardiol 66: 23-33, 2015.

16. Lev EI, Kornowski R, Vaknin-Assa H, Ben-Dor I, Brosh D, Teplitsky I, Fuchs S, Battler A and Assali A: Effect of previous treatment with statins on outcome of patients with ST-segment elevation myocardial infarction treated with primary percutaneous coronary intervention. Am J Cardiol 103: 165-169, 2009.

17. Ota T, Ishii H, Suzuki S, Tanaka A, Shibata Y, Tatami Y, Harata S, Shimbo Y, Takayama Y, Kawamura Y, et al: Relation between paradoxical decrease in high-density lipoprotein cholesterol levels after statin therapy and adverse cardiovascular events in patients with acute myocardial infarction. Am J Cardiol 115: 411-416, 2015.

18. Zheng X, Dreyer RP, Hu S, Spatz ES, Masoudi FA, Spertus JA, Nasir K, Li X, Li J, Wang S, et al: Age-specific gender differences in early mortality following ST-segment elevation myocardial infarction in China. Heart 101: 349-355, 2015.

19. Chen JW, Wang YL and Li HW: Elevated admission microalbuminuria predicts poor myocardial blood flow and 6-month mortality in ST-segment elevation myocardial infarction patients undergoing primary percutaneous coronary intervention. Clin Cardiol 35: 219-224, 2012.
20. Dong M, Mu N, Guo F, Zhang C, Ren F, Li J, Tao Z, Yang J and Li G: The beneficial effects of postconditioning on no-reflow phenomenon after percutaneous coronary intervention in patients with ST-elevation acute myocardial infarction. J Thromb Thrombolysis 38: 208-214, 2014.

21. Bonetti PO, Wilson SH, Rodriguez-Porcel M, HolmesDR Jr, Lerman LO and Lerman A: Simvastatin preserves myocardial perfusion and coronary microvascular permeability in experimental hypercholesterolemia independent of lipid lowering. J Am Coll Cardiol 40: 546-554, 2002.

22. Patti G, Pasceri V, Colonna G, Miglionico M, Fischetti D, Sardella G, Montinaro A and Di Sciascio G: Atorvastatin pretreatment improves outcomes in patients with acute coronary syndromes undergoing early percutaneous coronary intervention: Results of the ARMYDA-ACS randomized trial. J Am Coll Cardiol 49: 1272-1278, 2007.

23. Mandelzweig L, Battler A, Boyko V, Bueno H, Danchin N, Filippatos G, Gitt A, Hasdai D, Hasin Y, Marrugat J, et al: The second Euro Heart Survey on acute coronary syndromes: Characteristics, treatment, and outcome of patients with ACS in Europe and the Mediterranean Basin in 2004. Eur Heart J 27: 2285-2293, 2006.

24. Lee CH, Lee SH, Park JS, Kim YJ, Kim KS, Chae SC, Kim HS, Choi DJ, Cho MC, Rha SW, et al: Impact of statin usage patterns on outcomes after percutaneous coronary intervention in acute myocardial infarction: Korea Working Group on Myocardial Infarction registry (KorMI) study. J Geriatr Cardiol 11: 93-99, 2014. 